\title{
A New Interpretation of the Representational Theory of Measurement
}

\author{
Conrad Heilmann* \\ Forthcoming in Philosophy of Science
}

\begin{abstract}
On the received view, the Representational Theory of Measurement reduces measurement to the numerical representation of empirical relations. This account of measurement has been widely criticised. In this paper, I provide a new interpretation of the Representational Theory of Measurement that sidesteps these debates. I propose to view the Representational Theory of Measurement as a library of theorems that investigate the numerical representability of qualitative relations. Such theorems are useful tools for concept formation which, in turn, is one crucial aspect of measurement for a broad range of cases in linguistics, rational choice, metaphysics, and the social sciences.
\end{abstract}

\section{Introduction}

The Representational Theory of Measurement (RTM) is one of the main accounts of measurement (Swistak, 1990; Boumans, 2008; Cartwright and Chang, 2008). It characterises measurement as a mapping between two relational structures, an empirical one and a numerical one (Krantz et al., 1971; Suppes et al., 1989; Luce et al., 1990).

RTM is much criticised. Its critics, such as those that endorse a realist or operationalist conception of measurement, focus mainly on the fact that RTM advances an abstract conception of measurement that is not connected to empirical work as closely as it should be: it reduces measurement to representation, without specifying the actual process of measuring something, and problems like measurement error and the construction of reliable measurement instruments are ignored (Michell, 1990; Decoene et al., 1995; Michell, 1995; Boumans, 2007; Reiss, 2008).

\footnotetext{
${ }^{*}$ Erasmus Institute for Philosophy and Economics (EIPE), Faculty of Philosophy, Erasmus University Rotterdam, P.O. Box 1738, 3000 DR Rotterdam, The Netherlands, Email: heilmann@fwb.eur.nl
} 
In this paper, I do not engage with these worries, but rather sidestep them by proposing to interpret RTM in a different way. I will not assess RTM as a candidate theory of measurement, but propose the following two-step interpretation: firstly, RTM should be viewed as simply providing a library of mathematical theorems. Secondly, RTM theorems have a particular structure that makes them useful for investigating problems of concept formation. More precisely, I propose to view theorems in RTM as providing us with mathematical structures which, if sustained by specific conceptual interpretations, can provide insights into the possibilities and limits of representing concepts numerically. If we adopt this interpretation, there is no reason why RTM theorems should be restricted to specifying the conditions under which only empirical relations can be represented numerically. Rather, we can view the theorems as providing insights into how to numerically represent any sort of qualitative relation between any sort of object. Indeed, those objects can include highly idealised or hypothetical ones. On this view, RTM is no longer viewed as candidate for a full-fledged theory of measurement, but rather as a tool that can be used in discussing the formation of concepts, which in turn is often a particularly difficult part of measurement, especially in the social sciences.

This new interpretation of RTM has a number of advantages. Firstly, it allows us to use RTM theorems in the investigation of abstract concepts. All this means is that since we move from an empirical relational structure to a more general qualitative relational structure, we can also ask what kind of qualitative relations between imagined or idealised objects could be represented numerically. This is helpful in areas of inquiry in which there no developed empirical concepts and where there is a lack agreement on a number of basic questions (such as cases in linguistics, rational choice, metaphysics, and the social sciences more generally). Secondly, the new interpretation gives more flexibility to engage in 'backwards engineering' of foundations for quantitative concepts. In contexts in which we operate with numbers that lack adequate conceptual and epistemic foundations, we can investigate what kinds of qualitative relations between what kinds of objects would need to exist in order to motivate the kind of numbers that are already in use. That is, we can look at areas of inquiry that use quantities that are not derived from a measurement process and investigate whether these quantities can be seen as numerical representations of qualitative relations (and, in turn, whether such a representation can help in devising a measurement process). Thirdly, the interpretation serves as a way to rehabilitate RTM as a useful part of theoretical tools for measurement.

I proceed as follows. Section 2 introduces RTM in its received interpretation. Section 3 explains the new interpretation of RTM as representing qualitative instead of only empirical relations. Section 4 discusses desiderata of the interpretation. Section 5 briefly concludes. 


\section{The Representational Theory of Measurement}

Before RTM, measurement was mainly associated with the idea that (physical) quantities are assigned numbers (Russell, 1903, 176). RTM has taken a more abstract stance, substituting the idea of physical quantity or magnitude with properties or features of objects or with relations between such properties or features (Swistak, 1990). Swistak $(1990,7)$ also maintains that the 'representational paradigm is the fundamental notion of measurement which is in use in the contemporary theory of measurement' and ascribes the coining of the term 'representational theory of measurement' to Adams (1966). The authoritative statement of RTM can be found in the three books Krantz et al. (1971), Suppes et al. (1989) and Luce et al. (1990), which are building on earlier axiomatic work by Hölder, Helmhotz, Campbell, and others (for an overview, see Tal (2013)). In their characterisation, a representational measurement procedure allows one to make two formal statements,

'a representation theorem, which asserts the existence of a homomorphism $\phi$ into a particular numerical relational structure, and a uniqueness theorem, which sets forth the permissible transformations $\phi \mapsto \phi^{\prime}$ that also yield homomorphisms into the same numerical structure. A measurement procedure corresponds in the construction of a $\phi$ in the representation theorem.' (Krantz et al. $(1971,12)$.

In the received interpretation of RTM, we thus speak of a homomorphism between an empirical relational structure (ERS) and a numerical relational structure (NRS) characterising a measurement. For example, for simple length measurement, we might want to specify the ERS as $\langle X, \circ, \succcurlyeq\rangle$, where $X$ is a set of rods, $\circ$ is a concatenation operation, and $\succcurlyeq$ is a comparison of length of rods. If the concatenation and comparison of rods satisfy a number of conditions, there is a homomorphism into a $\mathrm{NRS}\langle\mathbb{R},+, \geq\rangle$, where $\mathbb{R}$ denotes the real numbers, + addition operations, and $\geq$ comparison operations between real numbers. As mentioned above, the existence of such homomorphism is asserted by a representation theorem.

The exact characterisation of what kind of scale a given measurement procedure yields is given by uniqueness theorems which specify the permissible transformations of the numbers. More formally, uniqueness theorems assert that ' . . . a transformation $\phi \mapsto \phi$ ' is permissible if and only if $\phi$ and $\phi^{\prime}$ are both homomorphisms ... into the same numerical structure ...' (Krantz et al., 1971, 12). Following Stevens (1946), a distinction is usually made between nominal, ordinal, interval and ratio scales. Nominal scales allow only for one-to-one transformations. Ordinal scales allow monotonic increasing transformations of the form $\phi \mapsto f(\phi)$. Interval scales allow for affine transformations of the form $\phi \mapsto \alpha \phi+$ 
$\beta, \alpha>0$. Ratio scales allow for multiplicative transformation of the form $\phi \mapsto \alpha \phi, \alpha>0$.

Particular variants of construction such scales have emerged, such as extensive, conjoint, bisection and difference measurement (reviewed in Suppes $(2002,63 \mathrm{ff}$.)). Representations in extensive measurement specify procedures that make use of the addition of magnitudes, such as in measuring physical magnitudes of mass and length. Bisection measurement gives representations by using the operation of identifying a midpoint in an interval. Conjoint measurement representations allow the combinations of magnitudes or properties, such as when measuring the intensity and frequency of a phenomenon. In difference measurement, representations capture the intensity of a particular property or relation. The three books by Krantz et al. (1971), Suppes et al. (1989) and Luce et al. (1990) contain a collection of mathematical results that pertain to these different measurements.

In the received interpretation, RTM takes measurement to consist in constructing homomorphisms of this kind:

'[...] measurement may be regarded as the construction of homomorphisms (scales) from empirical relational structures of interest into numerical relational structures that are useful.' (Krantz et al., 1971, 9)

I call this the received interpretation of RTM because on the one hand it is close to the sparse interpretative remarks given in what is now the authoritative statement of RTM, and on the other hand it also suggests that RTM constitutes a candidate theory of measurement.

RTM as a candidate theory of measurement has been met with a fair share of criticism in the literature. Since the purpose of this paper is to sidestep rather than to engage this criticism, I will not go into detail about it. The critics focus mainly on the fact that RTM advances an abstract conception of measurement that is not connected to empirical work as closely as it should be: it reduces measurement to representation, without specifying the actual process of measuring something, and problems like measurement error and the construction of reliable measurement instruments are ignored (Michell, 1990; Decoene et al., 1995; Michell, 1995; Boumans, 2007; Reiss, 2008). My proposed interpretation will sidestep these criticisms.

From this can be concluded that some critics regard RTM to be limited in serving as a theory of measurement. Where does this leave us with regards to RTM? I will not answer this question directly, as I will not assess the merits of RTM as a full-fledged theory of measurement in this paper. Rather, I argue in the following that a slightly modified interpretation of RTM can help to make it useful for a number of crucial exercises in several different fields. 


\section{Qualitative Relational Structures}

In this section, I outline the main elements of the new interpretation of RTM. The new interpretation proceeds in two simple moves: firstly, I start from viewing RTM as a library of mathematical theorems of a certain kind. Secondly, I change the standard interpretation of the domain of the representation theorems: instead of empirical relational structures, I will interpret them more generally as qualitative relational structures. With these two moves completed, we can use the theorems in RTM in a greater variety of contexts. Before elaborating on the latter, I will now explain the two moves in greater detail.

Firstly, RTM is simply viewed as a library of theorems. That is to say, in the following, the term RTM will refer to the theorems in the three books that contain the authoritative statement of RTM (Krantz et al., 1971; Suppes et al., 1989; Luce et al., 1990). Interestingly, there is relatively little by way of 'measurement' interpretation of the theorems in these three books, even though RTM is still considered to be one of the main theories of measurement, if not the dominant one (Boumans, 2008). The interpretation of the mathematical structures as referring to measurement is by and large confined to a few smaller sections in those three books (such as Chapter 1 of Krantz et al. (1971) and some sections of Chapter 22 in Luce et al. (1990)). More importantly, the idea that RTM is a full-fledged theory of measurement appears in the dozens of articles in which the different theorems have been initially been presented (extensively referenced in Krantz et al. (1971)). As perhaps the most poignant example of these articles, consider Davidson et al. (1955), in which we find extensive discussion of how the proposed theorems might make measurement in psychology and economics more 'scientific'. On the one hand, this suggests that the main proponents of RTM have undeniably intended it as a full-fledged theory of measurement. At the same time, the theorems in the three volumes cited above can also be seen separate from that. The first move of the new interpretation is to do just that and hence to consider RTM as the collection of mathematical theorems of a certain kind.

Secondly, from a mathematical point of view, the representation and uniqueness theorems in RTM simply characterise mappings between two kinds of structures, with one of these structures being associated with properties of numbers, and the other with qualitative relations. In the case of simple length measurement, the concatenation operation and the ordering relation are interpreted as actual comparisons between rods. Yet, since the theorem just concerns the conditions under which the concatenation operation and the ordering relation can be represented numerically, it is possible to furnish an even more general interpretation of what hitherto has been called ERS, the empirical relational structure. This more general interpretation is to replace the specific idea of ERS 
structure with that of a QRS, a qualitative relational structure.

Reinterpreting the empirical relational structure $\langle X, \circ, \succcurlyeq\rangle$ as a qualitative relational structure (QRS) does not require any change, addition or reconsideration of the measurement and uniqueness theorems in RTM. Indeed, all what is needed in order to apply the latter is that there is:

- a set of well specified objects in the mathematical sense: that we have clear membership conditions for the set $X$. Mathematically, RTM theorems do not require that the objects have empirical content.

- well-defined qualitative relations, such as $\circ$ and $\succcurlyeq$. Mathematically, RTM theorems do not require that these relations are interpreted empirically, i.e. that we can concatenate physical objects, or compare objects empirically.

The new interpretation of RTM hence sees it only as a collection of theorems that investigate how a QRS $\langle X, \circ, \succcurlyeq\rangle$ can be mapped into a NRS $\langle\mathbb{R},+, \geq\rangle$. It thus clearly sidesteps any of the criticisms of RTM in its received interpretation, since these criticisms were directed at RTM as a full-fledged theory of measurement, and focused on how RTM theorems apply to empirical relations.

\section{Advantages of the New Interpretation}

The new interpretation allows us to apply RTM theorems to any concept that we might care to investigate with regards to its potential for numerical representation. I will discuss two desiderata, firstly investigating the numerical representability of concepts, and secondly investigating possibilities of backwards engineering of foundations, before turning to defend the interpretation against two possible objections.

\subsection{Numerical Representability of Concepts}

With the new interpretation of RTM, we can also ask what kind of qualitative relations between imagined or idealised objects could be represented numerically. This is helpful in areas of inquiry in which there are no (or not yet developed) well-formed empirical concepts, and where there is a lack agreement on a number of basic questions.

Interpreting RTM theorems as specifying conditions of mappings between QRS and NRS, we can use them to speculate about possible numerical representations of abstract properties of abstract concepts. What is required for this are simply concepts that specify a well-defined set of objects and qualitative relations. There are some indications that RTM theorems are already used in such a way in different areas of inquiry. 
Take the case of linguistic analysis of interadjective comparisons (Bale, 2008). in this field, it is investigated how we can make sense of statements such as ' $x$ is $P$-er than $y$ is $Q$ ', with van Rooij (2011) applying RTM theorems to such statements to investigate whether properties $P$ and $Q$ of objects $x$ and $y$ are numerically representable, what possible scale properties such representations can fulfil, and hence in how far interadjective comparisons can be meaningful. In short, he uses RTM theorems to investigate to what extent abstract properties that are described by adjectives can be numerically represented, and in what way they can be compared.

Another case can be found in recent philosophical investigations concerning the foundations of rational choice. Traditionally, rational choice has used RTM-style theorems in their received interpretation, investigating how preferences can be represented numerically, under the assumptions that preferences are nothing but, or closely linked to, observable choice behaviour (Davidson et al., 1955). Yet, with cases of preference reversal and change, and investigations into how conflicting desires and beliefs can be captured by preferences, recent philosophical literature in rational choice theory has used RTM theorems without presupposing such close empirical links (see, for instance, Bradley (2009a), Bradley (2009b), Dietrich and List (2009), List and Dietrich (2013)). These articles investigate the determinants and changes of preferences by depicting them in an abstract way, leaving open how they are linked to observable or empirically testable entities or events.

These cases show, that some fields have already - unwittingly, or implicitly - adopted a more liberal interpretation of RTM theorems and tailored them to their needs. This suggests that the new interpretation of RTM fits well with scientific practice in some areas. At the same time, there are many more areas in which the new interpretation could help to structure similar exercises.

Consider, for instance the notorious case of personal identity over time in metaphysics (Noonan, 1989; Olson, 2002). As is well known, there is widespread disagreement over how to characterise personal identity over time, and the relevant literature is strewn with paradoxes and thought experiments that seem to pose insurmountable problems for any theory of personal identity over time. At the same time, these theories have undoubtably advanced our understanding of their subject. As a brief sketch how RTM theorems could be helping in further investigating theories of personal identity over time, consider Parfit (1984) who maintained to view persons as sets of temporal selves, and that personal identity consists in connectedness, which in turn is determined by an appropriate degree of psychological continuity between selves. To investigate to what extent the concept of a degree of psychological continuity can be represented numerically, we can interpret a QRS $\langle X, \circ, \succcurlyeq\rangle$ in which $X$ is a set of temporal selves, and $\circ$ and $\succcurlyeq$ are operations that join and compare the psychological continuity of selves. That is, we can imagine that there is 
a collection of temporal selves all of which might take differing attitudes, and who might overlap in various ways with each other. It is natural investigate these comparisons with RTM theorems: do they satisfy certain conditions such that the QRS of temporal selves and comparisons can be represented by some NRS? If so, we would be able to specify a concept of psychological continuity that is numerically representable.

Following the new interpretation of RTM is therefore both in line with recent developments in fields such as linguistics and rational choice, as well as open up applications of RTM theorems in other areas of inquiry.

\subsection{Backwards Engineering of Foundations}

A second advantage of the new interpretation of RTM is that it affords us greater flexibility in 'backwards engineering' of foundations. All this means is that in contexts in which we operate with numbers that lack adequate conceptual and epistemic foundations, we can investigate what kinds of qualitative relations between what kinds of objects would need to exist in order to motivate the kind of numbers that are already in use. That is, we can look at areas of inquiry that use quantities that are not derived from a measurement process and investigate whether these quantities can be seen as numerical representations of qualitative relations (and, in turn, whether such a representation can help in devising a measurement process). This holds especially for the social sciences, for which Cartwright (2008) already has made the case that RTM theorems can be very useful, even though she retained their received interpretation.

On the more general interpretation of RTM put forward here, we can jointly endorse both RTM and the view that there may be concepts relevant for a given area of inquiry that may not be directly observable. The new interpretation does allow for 'backwards engineering' of conceptual and epistemic foundations in different steps. Suppose there is some area of scientific inquiry in which numbers are currently used, yet there is ambiguity about how these numbers come about, i.e. what their conceptual foundations are and what they express, such as in happiness measurement and time discount rates in economics (and similar concepts in psychology, social science, and economics). The new interpretation of RTM allows firstly to use RTM theorems for conceptual clarification, for instance by asking in how far the concept of happiness can be represented numerically. If it is possible to conceive of the concept of happiness as numerically representable in a RTM theorem, then that is a key step in the investigation of the conceptual foundations. Secondly, we can also investigate epistemic (or evidential) foundations, by adopting the traditional interpretation of RTM, asking whether there are indeed empirical relations that sustain both the concept of happiness and a theorem in RTM.

As another example for backwards engineering of foundations, I highlight the con- 
tentious issue of time discounting in economics. The idea of discounting the future - to slightly devalue the utility of future events - was introduced into economic theory by a number of different authors, but most importantly by Ramsey (1928) and Samuelson $(1937,1939)$. These authors provided the idea of a discount rate with which future value would be weighted, which became common practice in economics. Only much later, the idea of discounting the future was investigated in a more thorough way, providing an axiomatic basis for it, notably by Koopmans (1960). The result of these developments is that time discounting is up until this day a contentious subject in economics, with many applications requiring the use of time discount rate, but with considerable ambiguity and controversy about descriptive and normative questions about time discounting remaining (see Frederick et al. (2002) for an overview). Put simply, most economic theorists and practitioners live by Ramsey's (1928) dictum that time discounting is 'a practice which is ... indefensible $[\ldots]$ we shall, however, ... include such a rate of discount in some of our investigations.'

From the point of view of the new interpretation of RTM, this practice can be seen as ascribing numbers (discount factors) to future prospects. Naturally, the question arises whether these numbers are meaningful, i.e. whether they correspond to quantities or empirical relations. However, since future prospects are not naturally empirical entities (they are, at best, propositions), RTM in its received interpretation would be inapplicable - or only applicable in as far as one can formulate future prospects as propositions that can be subjected to observable choice behaviour. Yet, many descriptive and normative questions about time discounting go beyond that, such as those that have to do with future generations, and those for which it is impossible to sensibly formulate choice-ready propositions, such as 'branching cases' considered in theories of personal identity over time.

In this context, RTM theorems have been used by some authors in both economics and philosophy, such as Fishburn and Rubinstein (1982); Ok and Masatlioglu (2007); Heilmann (2008), to investigate how far time discounting factors can be seen as numerical representations of concepts that are important about the future, such as impatience, different types of uncertainty, and ethical judgements of various kinds. Yet, most of these efforts have been bound by the received interpretation of finding corresponding empirical structures. Investigating hypothetical scenarios and comparisons between them is something that is only possible once the new interpretation of RTM is adopted.

More generally, any case in which one is confronted with the uses of numbers or the supposition of quantitative concepts can potentially investigated with the tools of RTM - if the new interpretation is adopted. 


\subsection{Objections}

I now turn to two objections against the new interpretation. Firstly, from the point of view of proponents of RTM as a full-fledged theory of measurement, the new interpretation might seem as 'giving in too quickly'. While it is true that the new interpretation does not endorse RTM as a full-fledged theory of measurement, it is not inconsistent with it. Rather, it spells out in what way RTM provides a useful tool, regardless of what general account of measurement they are invested in. Since numerical representability of concepts is a difficult part in many areas of measurement, rehabilitating RTM as a tool for those areas is a project that should appeal to the RTM supporter. Whether or not to additionally claim that RTM is useful beyond the two uses spelled out in the preceding sections is simply a different question that is independent from the new interpretation advanced here. Indeed, interpreting the relations as necessarily having empirical content is a special case of the more general interpretation I put forward here.

Secondly, it is possible to question whether the interpretation advanced here does make a big difference to RTM and its use. I think such an objection underestimates both the problems commonly associated with RTM in its received interpretation and their possible consequences. Since RTM is widely and heavily criticised as a theory of measurement (see Section 2), there is a real danger that it will be dispensed altogether. The consequence of that would, in turn, be that exercises as described in Section 4.1 and 4.2, already carried out in some fields, would be without an account that underpins them. Moreover, the additional perspectives to discuss the numerical representability of properties of hypothetical entities (such as temporal selves in metaphysics) or reasons to discount the future which cannot be easily grounded in empirical relations hang on adopting the new interpretation.

\section{Conclusions}

In this paper, I have proposed to interpret the Representational Theory of Measurement in a new way, namely as a library of theorems that investigate the numerical representability of qualitative relations. Such theorems are useful tools for concept formation which, in turn, can be seen as one crucial aspect of measurement for a broad range of cases in linguistics, rational choice, metaphysics, and the social sciences. I have suggested that it is already part of scientific practice to use RTM theorems in such a way, and have suggested that there are more cases to which they could be fruitfully be applied. 


\section{Acknowledgements}

Many thanks in particular to Eran Tal for many helpful comments, as well as to Constanze Binder, Marcel Boumans, Aki Lehtinen, Luca Mari, F.A. Muller, Julian Reiss, Jan-Willem Romeijn, and participants at the 2012 Arctic Workshop on Measurement in Rovaniemi and the 2013 OZSW Conference of the Dutch Research School of Philosophy in Rotterdam. Work on this article has been supported by a Marie Curie Career Integration Grant \#PCIG10-GA-2011-303900 from the European Union and a VENI grant \#275-20-044 from the Netherlands Organisation for Scientific Research (NWO).

\section{References}

Adams, E. W. (1966). On the nature and purpose of measurement. Synthese, 16, 125-169.

Bale, A. (2008). A universal scale of comparison. Linguistics and Philosophy, 31, 1-55.

Boumans, M. (2007). Measurement in Economics: A Handbook. AP Elsevier.

Boumans, M. (2008). Measurement. In S. N. Durlauf and L. E. Blume, editors, The New Palgrave Dictionary of Economics. Palgrave Macmillan, Basingstoke.

Bradley, R. (2009a). Becker's thesis and three models of preference change. Politics, Philosophy and Economics, 8(2), 223-242.

Bradley, R. (2009b). Preference kinematics. In T. Grüne-Yanoff and S. O. Hansson, editors, Preference Change, pages 221-242. Theory and Decision Library A, Springer.

Cartwright, N. (2008). In praise of the representation theorem. In M. Frauchiger and W. K. Essler, editors, Representation, Evidence, and Justification: Themes from Suppes, pages 83-90. Ontos Verlag.

Cartwright, N. D. and Chang, H. (2008). Measurement. In S. Psillos and M. Curd, editors, The Routledge Companion to Philosophy of Science, pages 367-375. New York: Routledge.

Davidson, D., McKinsey, J. C. C., and Suppes, P. (1955). Outlines of a formal theory of value, I. Philosophy of Science, 22(2), pp. 140-160.

Decoene, S., Onghena, P., and Janssen, R. (1995). Representationalism under attack. Review of An introduction to the logic of psychological measurement, by J. Michell and Philosophical and foundational issues in measurement theory, by C. Wade Savage and P. Ehrlich. Journal of Mathematical Psychology, 39(2), $234-242$. 
Dietrich, F. and List, C. (2009). A model of non-informational preference change. LSE Choice Group Working Paper Series, 5(1).

Fishburn, P. C. and Rubinstein, A. (1982). Time preference. International Economic Review, 23(3), 677-94.

Frederick, S., Loewenstein, G., and O'Donoghue, T. (2002). Time discounting and time preference: A critical review. Journal of Economic Literature, 40(2), 351-401.

Heilmann, C. (2008). Measurement-theoretic foundations of time discounting. LSE Choice Group Working Paper Series, 4.

Koopmans, T. (1960). Stationary ordinal utility and impatience. Econometrica, 28(2), 287-309.

Krantz, D. H., Luce, R. D., Tversky, A., and Suppes, P. (1971). Foundations of Measurement Volume I: Additive and Polynomial Representations. Mineola: Dover Publications.

List, C. and Dietrich, F. (2013). Where do preferences come from? International Journal of Game Theory, 42(3), 613-637.

Luce, R. D., Krantz, D. H., Tversky, A., and Suppes, P. (1990). Foundations of Measurement Volume III: Representation, Axiomatization, and Invariance. Mineola: Dover Publications.

Michell, J. (1990). An Introduction to the Logic of Psychological Measurement. Hillsdale, NJ: Erlbaum.

Michell, J. (1995). Further thoughts on realism, representationalism, and the Foundations of measurement theory. Author's Response to Review by Decoene et al. of An introduction to the logic of psychological measurement. Journal of Mathematical Psychology, 39(2), 243-247.

Noonan, H. W. (1989). Personal Identity. Routledge.

Ok, E. A. and Masatlioglu, Y. (2007). A theory of (relative) discounting. Journal of Economic Theory, 137, 214-45.

Olson (2002). Personal Identity. The Stanford Encyclopedia of Philosophy (Fall 2002 Edition), E. Zalta.

Parfit, D. (1984). Reasons and Persons. Clarendon. 
Ramsey, F. P. (1928). A mathematical theory of saving. Economic Journal, 38(152), $543-59$.

Reiss, J. (2008). Error in Economics: Towards a More Evidence-based Methodology. Routledge.

Russell, B. (1903). The Principles of mathematics. New York: Cambridge University Press.

Samuelson, P. (1937). A note on measurement of utility. Review of Economic Studies, 4, $155-61$.

Samuelson, P. (1939). The rate of interest under ideal conditions. The Quarterly Journal of Economics, 53(2), 286-97.

Stevens, S. S. (1946). On the theory of scales of measurement. Science, 103(2684), $677-680$.

Suppes, P. (2002). Representation and Invariance of Scientific Structures. Stanford: CSLI Publications.

Suppes, P., Krantz, D. H., Luce, R. D., and Tversky, A. (1989). Foundations of Measurement Volume II: Geometrical, Threshold, and Probabilistic Representations. Mineola: Dover Publications.

Swistak, P. (1990). Paradigms of measurement. Theory and Decision, 29(1), 1-17.

Tal, E. (2013). Old and new problems in philosophy of measurement. Philosophy Compass, 8(12), 1159-1173.

van Rooij, R. (2011). Measurement and interadjective comparison. Journal of Semantics, 28, 335-358. 